\title{
CALCULATING RADIAL TEXTURES IN BUSHES* MADE OF THE ALLOY Mn-Al-C UPON UPSETTING INTO A CONTAINER
}

\author{
V.D. SOLOVEI ${ }^{\dagger}$ and YU.N. LOGINOV \\ Institute of the Engineering Science, Urals State Technical University, \\ 91 Pervomaiskaja Str., 620219 Ekaterinburg GSP-207, Russia
}

(Received in final form 28 September 1997)

The alloy $\mathrm{Mn}-\mathrm{Al}-\mathrm{C}$ (magnetic $\tau$-phase) has a face-centered tetragonal crystal lattice with the superstructure $L 1_{0}$ (Shangurov et al., 1990). The tetragonal crystal axes $c$ (the directions $\langle 001\rangle$ of easy magnetization) of the grain lattices of a polycrystal permanent magnet having a form of a bush must be preferably oriented along the corresponding bush radius. Such bushes with a pronounced radial texture are used, for example, as stators of micromotors. In the present paper the forming of this radial texture $\langle 001\rangle$ in bushes made of the alloy $\mathrm{Mn}-\mathrm{Al}-\mathrm{C}$ is investigated theoretically. Texture inhomogeneity is taken into account by solving the boundary value problem of plasticity.

Keywords: Radial texture; Face-centered tetragonal lattice with the superstructure $L 1_{0}$; Slip and twin systems; Boundary value problem of plasticity; Particle texture perfection degree; Texture inhomogeneity

\section{INTRODUCTION}

The arbitrary grain lattice rotation increment vector on the strain increment can be represented by the equation (Taylor, 1938; Bunge, 1970; Van Houtte and Aernoudt, 1975)

$$
\mathrm{d} \omega_{i}=\mathrm{d} \bar{\omega}_{i}+\mathrm{d} \omega_{i}^{\mathrm{a}} .
$$

\footnotetext{
* Hereinafter the term bush stands for a hollow cylinder.

${ }^{\dagger}$ Corresponding author.
} 
The common rotation increment vector, $\mathrm{d} \bar{\omega}_{i}$, in the case of inhomogeneous strain, can be found from the boundary value problem of macroscopic plasticity.

In order to calculate the additional rotation increment vector, $\mathrm{d} \omega_{i}^{\mathrm{a}}$, it is necessary to know the grain strain increment for the particle strain step and the virtual shear systems for the grain lattice. According to Taylor's approximation (Taylor, 1938), the arbitrary grain strain increment is equal to particle strain increment. The shear systems acting in the grain are found with the help of Taylor's modified theory (Chin et al., 1969). Deformation of the alloy $\mathrm{Mn}-\mathrm{Al}-\mathrm{C}$ is accomplished by shears on the slip systems $\{111\}\langle 1 \overline{1} 0\rangle,\{111\}\langle 10 \overline{1}\rangle,\{111\}\langle 11 \overline{2}\rangle$ and twin systems $\{111\}\langle 11 \overline{2}\rangle$ (Shangurov et al., 1990). The present authors (Solovei and Loginov, 1996) suggest that the deformation textures in the alloy $\mathrm{Mn}-\mathrm{Al}-\mathrm{C}$ can be calculated only with the slip systems $\{111\}\langle 1 \overline{1} 0\rangle$, $\{111\}\langle 10 \overline{1}\rangle$ and the twin systems $\{111\}\langle 11 \overline{2}\rangle$. They also assume that the critical resolved stresses for all the slip systems are equal and that the critical resolved stresses for the twin and slip systems are connected by the relation $\tau_{\mathrm{t}}=0.6 \tau_{\mathrm{s}}$.

\section{YIELD KINEMATICS OF BUSHES UNDER UPSETTING INTO A CONTAINER}

The nonstationary process of bush upsetting into a container (see Fig. 1) can be considered as a quasi-stationary process. For this purpose, the value of any bush upsetting, $\varepsilon$, is divided into small upsetting steps $(\mathrm{d} h / h=0.01)$ and the deformation in each step is considered stationary. Small steps permit to decrease calculation errors connected with the use of quasi-stationary approximation and the problem of nonlinearity.

Bush yield kinematics on the upsetting step is found from the solution of the corresponding boundary value problem of Von Mises (1928) ideal plasticity.

Friction on the bush contact surfaces is neglected. In this case, the displacement increment vector components and the strain increment tensor components are represented by the following relations 


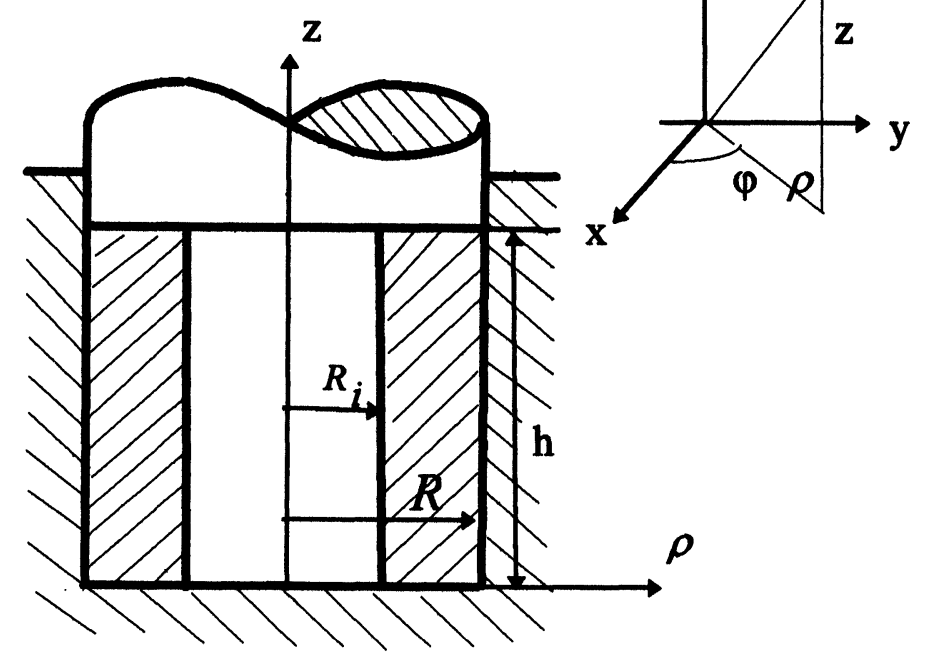

FIGURE 1 The scheme of bush upsetting into a container.

(Kolmogorov, 1970):

$$
\begin{aligned}
\mathrm{d} u_{r} & =\mathrm{d} h\left(R^{2}-\rho^{2}\right) / 2 h \rho, & \mathrm{d} u_{z}=-\mathrm{d} h z / h, \\
\mathrm{~d} \varepsilon_{r r} & =\mathrm{d} h\left(R^{2}+\rho^{2}\right) / 2 h \rho^{2}, & \mathrm{~d} \varepsilon_{z z}=-\mathrm{d} h / h, \\
\mathrm{~d} \varepsilon_{\varphi \varphi} & =-\mathrm{d} h\left(R^{2}-\rho^{2}\right) / 2 h \rho^{2} . &
\end{aligned}
$$

Hereinafter only non-zero components of the kinematic variables are mentioned. The common rotation increment vector components are zero.

From Eq. (2), the strain increments are seen to be independent of the coordinate $z$. It is seen that the dependencies of the components of the reduced strain increments $\mathrm{d} e_{r r}, \mathrm{~d} e_{\varphi \varphi}$ and $\mathrm{d} e_{z z}$ on the relative radius, $\bar{\rho}$ are the same for all the bush configurations and upsetting step values. These radial dependencies are shown in Fig. 2 (see Loginov et al., 1988). In the process under study, the strain increment tensor component $\mathrm{d} \varepsilon_{r r}$ is the principal lengthening increment $\mathrm{d} \varepsilon_{11}$ and the strain increment tensor components $\mathrm{d} \varepsilon_{\varphi \varphi}$ and $\mathrm{d} \varepsilon_{z z}$ are the principal shortening increments $\mathrm{d} \varepsilon_{22}$ 


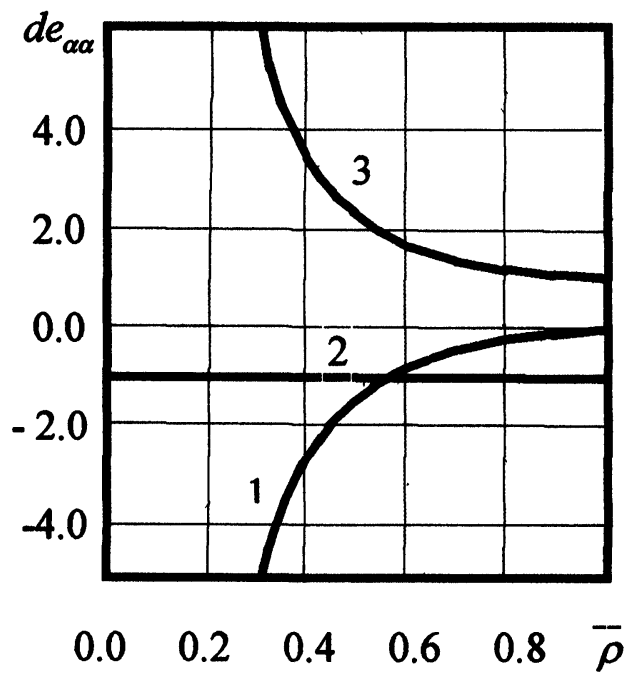

FIGURE 2 The dependencies of the components of the reduced strain increments, $\mathrm{d} e_{\alpha \alpha}(\alpha=r, \varphi, z)$ on the relative radius $\bar{\rho}$ for an arbitrary step of bush upsetting into a container: (1) $\alpha=\varphi$; (2) $\alpha=z$; (3) $\alpha=\rho$.

and $\mathrm{d} \varepsilon_{33}$. (The following relations take place: $\mathrm{d} \varepsilon_{11}>\mathrm{d} \varepsilon_{22} \geq \mathrm{d} \varepsilon_{33}$, $\mathrm{d} \varepsilon_{11}+\mathrm{d} \varepsilon_{22}+\mathrm{d} \varepsilon_{33}=0$.)

The characteristic section radius, $\rho^{*}$ is the same for all the bush sections and is described by the following equation:

$$
\bar{\rho}^{*} \equiv \rho^{*} / R=\sqrt{3} \approx 0.577 .
$$

It is seen from Fig. 2 that

$$
\begin{aligned}
& \mathrm{d} \varepsilon_{\varphi \varphi}=\mathrm{d} \varepsilon_{22}, \quad \mathrm{~d} \varepsilon_{z z}=\mathrm{d} \varepsilon_{33} \quad \text { for } \rho \geq \rho^{*} ; \\
& \mathrm{d} \varepsilon_{\varphi \varphi}=\mathrm{d} \varepsilon_{33}, \quad \mathrm{~d} \varepsilon_{z z}=\mathrm{d} \varepsilon_{22} \quad \text { for } \rho<\rho^{*} \text {. }
\end{aligned}
$$

\section{CALCULATED TEXTURES OF BUSHES MADE OF THE ALLOY Mn-Al-C UPON UPSETTING INTO A CONTAINER}

In the initial state, the bush has a random texture. The lattice orientation of an arbitrary grain of a deformed particle is determined by the initial 
grain orientation and by lattice rotation calculated as a sum of the lattice rotation increments (see Eq. (1)) on the small deformation path steps.

In the space of the Eulerian angles, the function of the grain lattice orientation distribution in the polycrystal particle, $g\left(\varphi_{1}, \vartheta, \varphi_{2}\right)$, is described discretely on the cells with the dimensions $45^{\circ}, 10^{\circ}$ and $45^{\circ}$ for the angles $\varphi_{1}, \vartheta$ and $\varphi_{2}$ respectively. The (particle) texture perfection degree, $S$, is found by the summation of this discrete distribution function over the grains with tetragonal axes lying in the $17^{\circ}$-interval near the corresponding radial direction of the bush.

Preliminarily, we study the process of particle texture formation in the system of principal strain directions. It is known (Shangurov et al., 1985) that the tetragonal axes of the grain lattices of the alloy $\mathrm{Mn}-\mathrm{Al}-\mathrm{C}$ rotate towards the direction of the principal lengthening and that an axial texture is formed in this direction. The texture perfection degree increases as the value of the principal lengthening, $\varepsilon_{11}$, increases. It was established experimentally (Shangurov et al., 1985) that, other things being equal, the texture perfection degree shows the most rapid increase when the values of the principal shortening, $\varepsilon_{22}$ and $\varepsilon_{33}$, are equal. The changes in the texture perfection degree for different deformation paths calculated in the space of the principal strains are shown in Fig. 3. It is evident that the dependencies shown describe the texture forming laws on the deformation steps in the system of principal strain increments.

The radial texture $\langle 001\rangle$ in the bush made of the alloy $\mathrm{Mn}-\mathrm{Al}-\mathrm{C}$ upset into a container is seen to be independent of the coordinate $z$ because the strain increment is independent of this coordinate. It is obvious from Figs. 2 and 3 that, for arbitrary bush deformation, the texture perfection degree on the internal bush surface must be greater than on the external one. This conclusion agrees with the known experimental data.

The present authors (Shangurov et al., 1985) studied the radial dependence of the magnetic properties of bushes made of the alloy $\mathrm{Mn}-\mathrm{Al}-\mathrm{C}$ obtained in the process under study. They deduced that the radial dependence of texture sharpness was extremal (nonmonotonic) for any values of the parameter $R_{\mathrm{i}}^{0} / R$. However, the texture perfection degree can be shown to be the extremal (nonmonotonic) function of the coordinate $\rho$ only for deformed bushes with $R_{\mathrm{i}}^{0}<\rho^{*}$. For this purpose, in the present paper two kinds of material lengths are considered. (The material length incorporates specific particles and it is deformed 


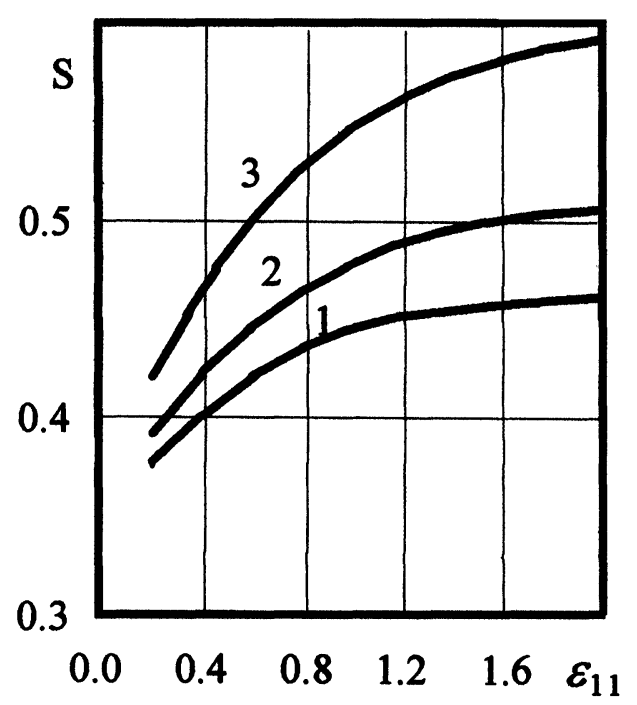

FIGURE 3 The dependence of the texture perfection degrees on the principal lengthening, $\varepsilon_{11}$ for some values of the principal shortenings, $\varepsilon_{22}$ and $\varepsilon_{33}$ : (1) $\varepsilon_{22}=0$; (2) $\varepsilon_{22}=\frac{1}{2} \varepsilon_{33} ;$; 3 ) $\varepsilon_{22}=\varepsilon_{33}$.

together with the bush.) In the initial (undeformed) state of the bush they are described by the following inequalities: $R_{\mathrm{i}}^{0} \leq \rho<\rho^{*}$ and $\rho^{*} \leq \rho \leq R$. It is evident that the bush has the material length of the second kind if $R_{\mathrm{i}}^{0} \geq \rho^{*}$ and that it has the material lengths of both kinds if $R_{\mathrm{i}}^{0}<\rho^{*}$. Taking into account Figs. 2 and 3, as well as the directions of the particle displacement in the deformation (towards the bush axis), we can conclude the following. For the material length of the second kind, in all the stages of bush deformation, a monotonic increase of the texture perfection degree towards the bush axis must take place. For a material length of the first kind, on small deformation from the initial bush state, the radial dependence of the texture perfection degree must be nonmonotonic because, in this case, the texture perfection degree increases as the particles within this length approach both its ends. This nonmonotony can take place in the increase of bush upsetting up to great values.

The radial dependencies of the texture perfection degree calculated for bushes having different values of the parameter $R_{\mathrm{i}}^{0} / R$ and upset up to the limit deformation which is obtained when the internal bush hollow disappears are presented in Fig. 4. The character of the curves shown corroborates the validity of the above-mentioned reasons. It is seen from 


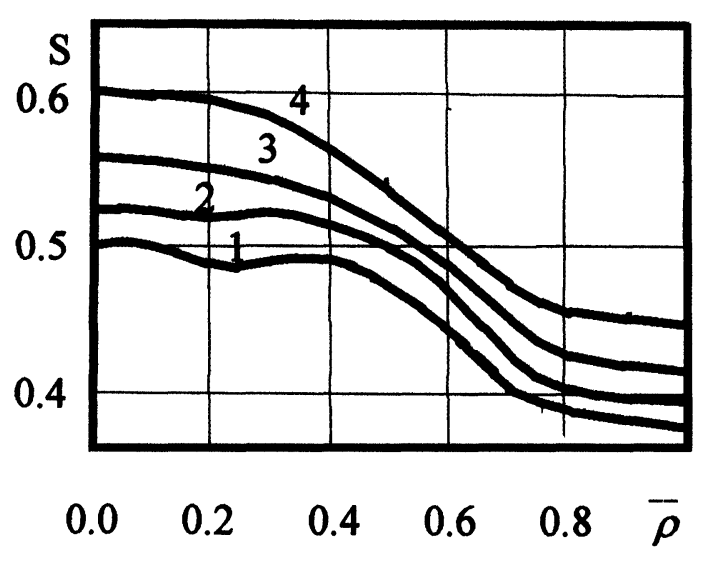

FIGURE 4 The radial dependencies of the texture perfection degrees in bushes upset up to the limit deformations (internal bush hollows disappear) for the following values of the parameter $R_{\mathrm{i}}^{0} / R$ : (1) $R_{\mathrm{i}}^{0} / R=0.4$; (2) $R_{\mathrm{i}}^{0} / R=0.5$; (3) $R_{\mathrm{i}}^{0} / R=0.6$; (4) $R_{\mathrm{i}}^{0} / R=0.8$.

this figure that the textures of all the bushes presented are substantially inhomogeneous. However, textures are more homogeneous in the regions bordering on the internal surfaces of the bushes (in the regions of the comparatively homogeneous texture). For the bushes with $R_{\mathrm{i}}^{0} \geq \rho^{*}$, the textures are sharper and the regions of comparatively homogeneous textures are smaller as compared with the case when $R_{\mathrm{i}}^{0}<\rho^{*}$.

The laws discovered here can be applied to the production of permanent magnets with required texture sharpness and homogeneity. For example, compound bushes can be upset and the internal elements of these bushes can be used as desired magnets.

\section{CONCLUSION}

The formation or radial texture $\langle 001\rangle$ in bushes made of the alloy $\mathrm{Mn}-\mathrm{Al}-\mathrm{C}$ upon upsetting into a container is investigated with the application of the deformation texture theory, with consideration for crystallographic slipping and twinning. Texture inhomogeneity in the radial direction is studied by means of the boundary value problem for continuum mechanics. The results obtained can be used to optimize for the texture (sharpness and homogeneity) of the bushes in the process under study. 


\section{NOTATION}

\begin{tabular}{|c|c|}
\hline$x, y, z$ & Cartesian coordinates \\
\hline$\rho, \varphi, z$ & cylindrical coordinates \\
\hline$R$ & external radius of the bush \\
\hline$h^{0}$ and $R_{\mathrm{i}}^{0}$ & $\begin{array}{l}\text { height and internal radius of the bush in the initial } \\
\text { (undeformed) state }\end{array}$ \\
\hline$h$ and $R_{\mathrm{i}}$ & $\begin{array}{l}\text { height and internal radius of the bush in the } \\
\text { deformed state }\end{array}$ \\
\hline $\bar{\rho}=\rho / R$ & relative radius \\
\hline$\varepsilon=\ln \left(h^{0} / h\right)$ & bush upsetting \\
\hline $\mathrm{d} h / h$ & bush upsetting step \\
\hline $\mathrm{d} u_{\alpha}$ & displacement increment vector \\
\hline $\mathrm{d} \varepsilon_{\alpha \beta}$ & (plastic) strain increment tensor \\
\hline $\mathrm{d} \varepsilon_{11}, \mathrm{~d} \varepsilon_{22}, \mathrm{~d} \varepsilon_{33}$ & principal strain increments \\
\hline $\mathrm{d} e_{\alpha \beta}=\mathrm{d} \varepsilon_{\alpha \beta} h / \mathrm{d} h$ & reduced strain increment tensor \\
\hline$\varepsilon_{11}, \varepsilon_{22}, \varepsilon_{33}$ & principal strains \\
\hline $\mathrm{d} \omega_{\alpha}$ & grain lattice rotation increment vector \\
\hline $\mathrm{d} \bar{\omega}_{\alpha}$ & $\begin{array}{l}\text { common rotation increment vector (refers to } \\
\text { particles) }\end{array}$ \\
\hline $\mathrm{d} \omega_{\beta}^{\alpha}$ & $\begin{array}{l}\text { additional rotation increment vector connected } \\
\text { with the acting shear (slip and twin) systems in the } \\
\text { grain (refers to grain lattices) }\end{array}$ \\
\hline$\tau_{\mathrm{s}}$ and $\tau_{\mathrm{t}}$ & $\begin{array}{l}\text { critical resolved stresses for the slip and twin systems } \\
\text { respectively }\end{array}$ \\
\hline$\rho^{*}$ & $\begin{array}{l}\text { characteristic radius of the bush section determined } \\
\text { as the coordinate } \rho \text { of the characteristic point where } \\
\text { two principal shortening increments are equal }\end{array}$ \\
\hline$S$ & $\begin{array}{l}\text { particle texture perfection degree determined as the } \\
\text { relative volume of all the particle grains with tet- } \\
\text { ragonal axes lying in a } 17^{\circ} \text {-interval near the radial } \\
\text { direction }\end{array}$ \\
\hline
\end{tabular}

\section{References}

Bunge, H.J. (1970). Some application of the Taylor theory of polycrystal plasticity. Kristall und Technik, 5, 145-175.

Chin, G.Y., Hosford, W.F. and Mendorf, D.R. (1969). Accomodation of constrained deformation in f.c.c. metals by slip and twinning. Proceeding of the Royal Society, A, $309,433-456$. 
Kolmogorov, V.L. (1970). Stresses, strains, fracture. M.: Metallurgiya, 229 S (in Russian).

Loginov, Yu.N., Burkin, S.P. and Shchipanov, A.A. (1988). The study of the strain state of a hollow cylinder upset into a container. Dep.v VINITI, No.7111-V88 ot 23.09.88, $14 \mathrm{~S}$ (in Russian).

Mises, R.V. (1928). Mechanik der plastischen Formänderung von Kristallen. Zeitschrift fur angewandte Mathematik und Mechanik, 8, 161-185.

Shangurov, A.V., Teitel, E.I., Yermakov, A.E. and Uimin, M.A. (1985). Effect of the strain state on the magnetic texture of the alloys $\mathrm{Mn}-\mathrm{Al}-\mathrm{C}$. Fizika metallovi metallovedenie, 6, 1171-1176 (in Russian).

Shangurov, A.V., Gornostyrev, Yu.N., Teitel, E.I., Gundyrev, V.M., Yermakov, A.E. and Solovei, V.D. (1990). Texture forming during cold deformation of the alloy $\mathrm{Mn}-\mathrm{Al}-\mathrm{C}$ ordered similarly to the type $L 1_{0}$. Fizika metallov $i$ metallovedenie, 6, 95-101 (in Russian).

Solovei, V.D. and Loginov, Yu.N. (1996). Calculating extrusion textures of the alloy $\mathrm{Mn}-\mathrm{Al}-\mathrm{C}$. Textures and Microstructures, 28, 121-128.

Taylor, G.I. (1938). Plastic strain in metals. Journal of the Institute of Metals, 62, 307-324.

Van Houtte, P. and Aernoudt, E. (1975). Solution of the generalized Taylor theory of plastic flow. Part 2. Zeitschrift für Metallkunde, 66, 202-209. 